\title{
Microbial community composition associated with early developmental stages of the Indian white shrimp, Penaeus indicus
}

Vinay TN ( $\sim$ vinaytn56@gmail.com )

ICAR - Central Institute of Brackishwater Aquaculture https://orcid.org/0000-0001-6863-1552

\section{Patil PK}

ICAR - Central Institute of Brackishwater Aquaculture

\section{Aravind $\mathrm{R}$}

ICAR - Central Institute of Brackishwater Aquaculture

Shyne Anand PS

ICAR - Central Institute of Brackishwater Aquaculture

\section{Baskaran V}

ICAR - Central Institute of Brackishwater Aquaculture

\section{Balasubramanian CP}

ICAR - Central Institute of Brackishwater Aquaculture

\section{Research Article}

Keywords: Aquaculture, Indian white shrimp, P. indicus, Microbiota, Early developmental stage, MOTHUR

Posted Date: December 28th, 2021

DOI: https://doi.org/10.21203/rs.3.rs-1203042/v1

License: (9) (i) This work is licensed under a Creative Commons Attribution 4.0 International License. Read Full License 


\section{Abstract}

Gut microbiota is known to influence the physiology, health, nutrient absorption, reproduction, and other metabolic activities of aquatic organisms. Microbial composition can influence intestinal immunity and are considered as health indicators. Information on gut microbial composition provides potential application possibilities to improve shrimp health and production. In the absence of such information for Penaeus indicus, the present study reports the microbial community structure associated with its early developmental stages. Bacterial community associated with the early developmental stages (egg, nauplii, zoea, mysis, postlarvae-1, postlarvae- 6 and postlarvae-12) from two hatchery cycles were analysed employing 16S rRNA high throughput sequencing. Proteobacteria and Bacteroidetes, were the two dominant phyla in $P$. indicus development stages. Sequential sampling revealed the constant change in the bacterial composition at genus level. Alteromonas was dominant in egg and nauplii stage, whilst Ascidiaceihabitans (formerly Roseobacter) was the dominant genera in both PL6 and PL12. The bacterial composition was highly dynamic in early stages and our study suggests that the mysis stage is the critical phase in transforming the microbial composition and it gets stabalised by early post larval stages. This is the first report on the composition of microbiota in early developmental stages of $P$. indicus. Based on these results the formation of microbial composition seems to be influenced by feeding at early stages. The study provides valuable information to device intervention strategies for healthy seed production.

\section{Introduction}

Complex symbiotic microbiota inhabits the intestine and is known to play significant role in the host physiology, health, nutrient absorption, reproduction, and other metabolic activities in terrestrial and aquatic animals [1-6]. Diversity of gut bacterial communities shift significantly during early growth stages and stabilizes later [7], and the abundance of certain group of bacteria can be considered as "health or disease indicators" [8]. Studies characterizing the colonization of gut microbiota in aquatic animals $[7,9,10]$, and the influence of a bacterial community or an individual bacterial species, on intestinal immunity $[1,2,11]$ have been reported recently. However, very few have attempted to describe the dynamics of microbes in the early developmental stages including egg, nauplii, zoea, mysis and early post larvae of shrimp when they are vulnerable to diseases.

Information on microbiota provides potential application possibilities to improve the production of food animals. Recent studies have reported the formation of microbiota, which get stabilized by post larval stages (PL5-PL15), indicating the role of early feeding, food and feeding habits, feed additives in shaping the gut microbiome in penaeid shrimp $P$. monodon [9] and $P$. vannamei [12]. Recently, we have reported the gut microbial composition in adult $P$. indicus obtained from wild and farmed environments [13]. However, information on the formation of microbiota in the early developmental stages of Indian white shrimp, $P$. indicus is not available. 
Shrimp farming is a major aquaculture activity in several south-Asian and south American nations including India [14, 15]. P. indicus is endemic to Indo-West Pacific and Middle Eastern regions [16] and suggested as an indigenous complimentary species [17] alongside $P$. vannamei, a globally dominant aquaculture species. Infections due to bacterial pathogens is considered as a major challenge in $P$. indicus seed production in hatcheries requiring scientific intervention for economical sustainability. Understanding the dynamics of establishment of species-specific microbiota will help in devising intervention strategies to produce healthy shrimp seeds. The present study reports the investigation of gut microbial communities from early developmental stages (egg to post larva) of $P$. indicus using 16S rRNA high throughput sequencing.

\section{Materials And Methods}

\section{Shrimp seed production and sampling}

Indian white shrimp (Penaeus indicus) larvae were produced in two full-sib batches at Muttukadu Experimental Station of ICAR-Central Institute of Brackishwater Aquaculture, Chennai, India. Healthy brooders were shifted to spawning room and maintained in UV sterilized seawater (35 ppt) in 250L tanks. Post spawning, the eggs were disinfected (Povidone-iodine $100 \mathrm{ppm}$ ) and allowed to hatch in $500 \mathrm{~L}$ tanks. After hatching, the healthy phototactic nauplius was collected and stocked in larval rearing tanks (100 no/L). The first feeding was started from zoea- 1 stage with live Thalassiosira sp. $(40-80,000 \mathrm{cell} / \mathrm{s} / \mathrm{ml})$ till post larvae. The post-larval stages were fed with brine shrimp nauplius (Artemia) at 4-5 no/PL. Ten precent water exchange was carried out daily from mysis stage and no antibiotic, probiotic was added throughout the study. Samples from both the batches were collected at egg, nauplii, zoea (Z2), mysis (M2), PL1, PL6 and PL12 stages (Fig. 1). Due to the small size of shrimp larvae, and to avoid individual bias, pooled samples (Egg: 200; Nauplii: 200; Zoea: 100; Mysis: 100; PL1: 50; PL6: 50; PL12: 25) were collected and were washed in sterile $\mathrm{NaCl}$ solution for 2-3 minutes and processed immediately for DNA extraction in both the batches.

DNA extraction, amplification, and sequencing of $16 S$ rRNA (V3-V4 region)

Whole shrimp pooled larval samples (Approximately: Egg: 200; Nauplii: 200; Zoea: 100; Mysis: 100; PL1: 50; PL6: 50; PL12: 25) were homogenised using sterile pestle for DNA extraction. Genomic DNA was extracted using the QIAamp DNA stool mini kit (Qiagen, Germany) following the manufacturer's protocol. The DNA concentration and purity were determined using NanoDrop ND-1000 spectrophotometer (Thermo Scientific, USA) and stored at $-80^{\circ} \mathrm{C}$ until used.

The amplicon libraries were prepared using the Nextera XT index kit (Illumina Inc. USA) as per metagenomic sequencing library preparation protocol. Amplification of bacterial 16S rRNA gene was carried out using the primers F GCCTACGGGNGGCWGCAG and R ACTACHVGGGTATCTAATCC. The amplicon libraries were purified by AMPPure XP beads and quantified using Qubit Flurometer. Library QC was checked on Agilent 4200 Tape station system and sequenced using MiSeq platform. 


\section{Amplicon sequence processing and microbiome analysis}

The raw sequences were processed using MOTHUR pipeline (v. 1.42) [18] to filter reads, create contigs; reduce noise as per standard MiSeq procedure. Sequences were aligned, clustered, and identified taxonomically with SILVA database (http://arb-silva.de) release 138. Chimera.vsearch option was used to identify and remove chimeras. Sequences with $97 \%$ identity threshold were classified into operational taxonomic units (OTUs) at genetic distances of 0.03 . Alpha diversity indexes were calculated from rarefied samples using calculators for richness and diversity indices of the bacterial community (i.e., Chao, ACE, and Shannon). Statistically significant correlation with the coordinates was tested for the PCoA plot $(p<0.05)$. Venn diagram was prepared using a web-based tool, interactiVenn [19]. Nonparametric t-test was carried out using linear discriminant analysis (LEfSe) to determine the significantly differing OTU's between the groups. The data output was subjected to detailed statistical and metaanalysis using MicrobiomeAnalyst [20] and Microsoft Excel for visualisation.

\section{Data availability}

The datasets of $16 \mathrm{~S}$ rRNA amplicon sequences obtained in this study were submitted to the NCBI BioProject ID (PRJNA718452).

\section{Results}

\section{Overview of sequencing analysis}

A total of 2541888 reads were obtained from 14 samples, with mean reads of 181563 per sample. A total of 1221 OTU's were obtained in the study. Good's coverage of $>99 \%$, indicated the sufficient sequencing depth to represent all the bacterial communities. Alpha-diversity indices (Ace, Chao 1, and Shannon) of different developmental stages of Indian white shrimp were calculated. Ace and Chao 1 are richness indicators, which measures different kinds of organisms present. The richness index Chao1 varied from $579.86 \pm 22.25$ to $841.22 \pm 16.82$ and ACE varied from $564.56 \pm 11.72$ to $828.70 \pm 14.17$. Our observations suggested that the richness of microbes steadily increased from egg till mysis and gradually stabalised in post larval stages though there was an increase in PL12 stage. However, Shannon is a diversity indicator, which takes into account the richness of the microbes and how evenly they are distributed. The diversity index Shannon showed that the mysis stage was more diverse and PL12 was less diverse inspite of having increased richness (Table 1).

\section{Bacterial diversity and composition}

P. indicus developmental stages microbiome contained a diverse community composition characterized into 21, 34, 29, 29, 27, 30 and 33 phyla, which were further classified to 243, 586, 584, 542, 457, 491 and 566 genera for egg, nauplii, zoea, mysis, PL1, PL6 and PL12 respectively (Table S1). The major phyla associated with the early developmental stages of $P$. indicus were Proteobacteria, Bacteroidetes, Actinobacterota, Firmicutes, Planctomycetes and 
Verrucomicrobiota. Proteobacteria and Bacteroidetes were the top two dominant bacterial phyla in all the developmental stages of the shrimp and the rest varied in the abundance and prevalence (Fig. 2).

The Proteobacteria was the most dominating phylum in all the developmental stages studied. Nearly half of the phyla belong to Proteobacteria till larvae reach mysis stage, which further increased to more than $60 \%$ during the PL stages. Bacteroidetes was the second most dominating between $22-35 \%$ in the early stages, which further reduced to about $10 \%$ during the late post larval stages (Table 2 ).

\section{Bacterial community associated with different developmental stages}

The most abundant genera from the most abundant phyla (Proteobacteria, Bacteroidetes, Actinobacteria, and Firmicutes) were compared in all the developmental stages (Fig. 3). Alteromonas was the most dominant genera in egg (19.67\%) and nauplii (12.55\%). Winogradskyella (12.64\%), Mesoflavibacter (10.97\%), Haloferula (5.23\%) and Rhodobacteraceae_unclassified (4.61\%) were dominant in egg; while Nautella (9.02\%), Tenacibaculum (5.94\%), Vibrio (5.64\%) and Pseudoalteromonas (5.42\%) were observed in nauplii. The top five bacterial genera in zoea stage were Roseibacillus (12.85\%), Saprospiraceae_unclassified (6.92\%), Rubinisphaeraceae_unclassified (6.73\%), Rhodobacteraceae_unclassified (6.44\%) and uncultured (5.29\%); while uncultured (8.17\%), NS3a_marine_group (6.59\%), Pseudoalteromonas (6.40\%), Rhodobacteraceae_unclassified (5.90\%), Kordiimonas (5.24\%) were dominant in mysis. Rhodobacteraceae_unclassified was found to be common dominant genera between zoea and mysis. In PL1, Vibrionaceae_unclassified (14.77\%), Kordia (11.01\%), Lactobacillus (3.60\%), Vibrio (7.35\%), Pseudomonas (6\%) were present. Pseudomonas started increasing in postlarvae stages (PL1 (6\%), PL6 (13.51\%) and PL12 (13.05\%); while Ascidiaceihabitans was common in PL6 (19.95\%) and PL12 (19.05\%), Gammaproteobacteria_unclassified (PL6: 14.01\%, PL12: 11.20\%) and Pseudarthrobacter (PL6: 4.19\%, PL12: 6.01\%) were also observed to be common between PL6 and PL12 (Fig. 4, Table 2).

\section{Comparison of the bacterial community structures}

Principal coordinate analysis (PCoA) based on the Bray-Curtis distance was used at the feature level to compare the composition of bacteria associated with $P$. indicus at early developmental stages (Fig. 5). The bacterial communities associated with shrimp were different across all developmental stages. However, the bacterial profiles in PL6 and PL12 were not significantly different as observed in $\beta$ diversity analysis. The results indicate that the bacterial compositions became stable once they enter post larval stage. PERMANOVA analysis, confirm that bacterial profiles were strongly associated with shrimp developmental stages (F-value: 2.1962; R-squared: 0.65308; p-value <0.005).

\section{Shared bacterial community associated with shrimp at early life stages}

Venn diagram showed 70 shared genera that were associated with all the developmental stages (Fig. 6). The shared genera were classified to phyla Proteobacteria, Bacteroidetes, Planctomycetes, Actinobacteria, 
Firmicutes, Cyanobacteria, Chloroflexi and Bdellovibrionota mainly dominated by Proteobacteria. Further, unique genera specific to egg (6), nauplii (65), zoea (44), mysis (30), PL1 (17) and PL12 (57) were observed in the study. Linear discriminant analysis effect size (LEfSe) was performed to identify the specific taxa significantly varied in abundance in early developmental stages. In total, 34 genera, varying significantly were identified with LDA scores $>4$ (Fig. S1).

\section{Discussion}

It is increasingly evident that host-microbiota interaction plays a major role in growth and developmental stages of both terrestrial and aquatic animals. Co-evolution of host and microbes has always been overlooked during the evolution of aquatic organisms [10]. Further, it was always considered that the embryo/ egg is sterile and has no microbiota. However, with the advancement of technology, the presence of bacteria in the eggs of Cod and Halibut was reported previously [21] and it is well understood that the microbiota is transferred vertically and horizontally. Recent studies in P. monodon [9], P. vannamei [8, 12], Macrobrachium rosenbergii [10]; Procambarus clarkii [22], Panulirus ornatus [23] have shown the presence of microbiota in the eggs and early developmental stages of these aquatic crustaceans. Understanding these host-microbial community relationship will help in devising intervention strategies for healthy shrimp production. Here, we report the microbiota associated with the early developmental stages of $P$. indicus for the first time employing high throughput sequence analysis.

Analysis of $16 \mathrm{~S}$ rRNA remains the standard culture-independent approach to investigate microbial diversity [24]. Alpha diversity is a measure of compositional complexity within an ecosystem. Ace and Chao 1 are richness indicators, which measures different kinds of organisms present. While, Shannon is diversity indicator, which takes into account the richness of the microbes and how evenly they are distributed. Our observations suggested that the richness of microbes steadily increased from egg till mysis and gradually stabalised in post larval stages though there was an increase in PL12 stage. The diversity index Shannon showed that the mysis stage was more diverse and PL12 was less diverse inspite of having increased richness. The observed richness in PL12 might be due to the presence of some rare microbes which are not evenly distributed. While, the zoea and mysis stages was highly diverse, which might be due to the initiation of feeding during these stages. Several studies tracking gut microbial composition during developmental stages have implicated the feeding to be the cause of bacterial community changes $[1,9,12]$. PCoA plot showed that samples belonging to PL6 and PL12 from full sib families clustered together indicating similar diversity. Interestingly, the bacterial profile at each developmental stage varied between the families as the growth progress. Further studies with large number of full-sib batches and multiple replicates could explain the influence of family and individual on bacterial profile.

The present study identified 70 shared genera, classified to phyla Proteobacteria, Bacteroidetes, Planctomycetes, Actinobacteria, Firmicutes, Cyanobacteria, Chloroflexi and Bdellovibrionota, during the developmental stages of $P$. indicus. Proteobacteria and Bacteroidetes were the top two dominant bacterial phyla associated with all the developmental stages, while Actinobacterota, Firmicutes, 
Planctomycetes and Verrucomicrobiota varied in prevalence and abundance.

Proteobacteria and Bacteroidetes are considered as resident microbiota of several aquatic organisms such as Neocaridina denticulate [25], P. chinensis [26], P. vannamei [12], P. monodon [27-29]. Angthong et al. [9], Liu et al. [10] and Wang et al. [12] reported that Proteobacteria and Bacteroidetes are the dominant phyla in the early developmental stages in in $P$. monodon, $M$. rosenbergii and $P$. vannamei respectively in agreement with the present study.

Each developmental stages showed the dominance of different microflora indicating the role of host factors in shaping the microbiota of an organism. Alteromonas (19.67\% and $12.55 \%$ ) were the major bacterial genera in egg and nauplii, which diminished in later stages. The symbiotic association of Alteromonas sp with the embryo of marine crustacean Palaemon macrodactylus, protecting from fungal infection by producing antifungal metabolite was reported [30]. The dominance of genus Alteromonas in the egg and nauplii of $P$. indicus suggest similar role during the developmental stage. Further, Vibrio was observed in all the stages, but was abundant in PL1 stage. Studies have shown that Vibrio is commonly associated with several aquatic organisms and our observations agree with the observations of Angthong et al. [9] in the developmental stages of $P$. monodon. Initiation of feeding during the zoea and mysis stages may be the reason for the observed colonisation by several group of microbes without being dominated by any specific group. Ascidiaceihabitans (formerly Roseobacter) was the dominant genera in both PL6 and PL12. Roseobacter are reported to be associated with the gut of several aquatic organisms and have essential metabolic capabilities to utilize proteins [31, 32], which help in growth of an organism. Recently, several species of Roseobacter are reported to have probiotic activity [33].

The dominant association of genera Winogradskyella and Mesoflavibacter under phylum Bacteroidetes with egg stage was observed, however their role in the development of larvae is not known.

Tenacibaculum is normally associated with the marine algae [34] and the presence of these genera in various stages of shrimp development may be associated with the planktonic feed provided in the hatchery. Kordia was dominant during PL1 stage, and it is known to utilize polysaccharides originated by marine phytoplankton or algae [35] and it is reported to increase in the presence of algal bloom and utilize the organic matter. Further, the genus under Actinobacterota, Firmicutes, Planctomycetes and Verrucomicrobiota were also reported. In a similar study in M. rosenbergii, strong association of Proteobacteria, Bacteroidetes and Actinobacterota was observed [10], while Planctomycetes and Verrucomicrobiota were present in relation to feeding and environment. Similarly, the shift in the microbial dynamics at different life stages has been reported from other invertebrates like butterfly, silkworm [36, 37]. Our study suggests that the mysis stage is the critical phase in transforming the microbial composition and gets stabalised by post larval stages. During the early stage, shrimp nauplii develop using yolk. The possible entry of microbial community associated with early feeding in addition to other metabolic activities in the gut might be the reason for the observed changes in the bacterial community. Similar observation in $P$. monodon was suggested to be due to the influence of host physiology and diets [9]. Further studies including a greater number of replicates are required for enhanced resolution of the bacterial diversity specific to each developmental stage of the species. 
To our knowledge this is the first report on the characterization of microbiota associated with early developmental stages of $P$. indicus. The bacterial composition was highly dynamic, and stage specific dominance of genera was observed. Modulation of gut microbial composition through supplementation of beneficial microbes at the mysis stage might improve survival and help in healthy and disease-free shrimp seed production. Further studies including multiple families is required to confirm the influence of external feeding on the microbial diversity.

\section{Declarations}

\section{Acknowledgements}

Authors acknowledge the facilities and support provided by the Director, ICAR-CIBA to carry out this work through the institute funded project FISHCIBASIL201800200130 Consortia Research Platform (CRP) on Vaccine and Diagnostics (CRP-V\&D) 1007611.

\section{Author contributions:}

C.P.B, P.K.P, and V.T.N: planning of the study, P.K.P: critical evaluation and fund mobility. V.T.N, A.R, and S.A.P.S: Shrimp seed production and larval rearing, V.T.N and V.B: Sampling and Sequencing. V.T.N: Metagenomic analysis and data interpretation. P.K.P and V.T.N wrote the manuscript, C.P.B: reviewed and provided valuable inputs to the manuscript. All authors have read and approved the final manuscript.

Availability of Data and Material: The original contributions presented in the study are included in the article/Supplementary Information; further inquiries can be directed to the corresponding author.

Code Availability: Not applicable.

Ethics approval: Not applicable.

Consent to participate: Not applicable.

Consent for publication: Not applicable.

Conflict of interest: The authors declare that the research was conducted in the absence of any commercial or financial relationships that could be construed as a potential conflict of interest.

\section{References}

1. Holt CC, Bass D, Stentiford GD, Giezen MVD (2020) Understanding the role of the shrimp gut microbiome in health and disease. $\mathrm{J}$ Invert Pathol 21:107387

2. Rajeev R, Adithya KK, Kiran GS, Selvin J (2021) Healthy microbiome: A key to successful and sustainable shrimp aquaculture. Rev Aquacult 13:238-258 
3. Butt RL, Volkoff H (2019) Gut microbiota and energy homeostasis in fish. Front Endocrinol 10:9

4. Kho ZY, Lal SK (2018) The human gut microbiome-A potential controller of wellness and diseases. Front Microbiol 9:1835

5. Li E, Xu C, Wang X, Wang S, Zhao Q, Zhang Z, Qin GJ, Chen L (2018) Gut microbiota and its modulation for healthy farming of Pacific White Shrimp Litopenaeus vannamei. Rev Fish Sci Aquacult 26:381-399

6. Nelson TM, Rogers TL, Brown MV (2013) The gut bacterial community of mammals from marine and terrestrial habitats. PLoS ONE 8(12):e83655

7. Stephens WZ, Burns AR, Keaton S, Sandi W, John FR, Karen G, Brendan JMB (2016) The composition of the zebrafish intestinal microbial community varies across development. The ISME J 10:644-654

8. Zheng Y, Min Y, Liu J, Qiao Y, Wang L, Li Z, Zhang XH, Yu M (2017) Bacterial community associated with healthy and diseased Pacific white shrimp (Litopenaeus vannamei) larvae and rearing water across different growth stages. Front Microbiol 8:1362

9. Angthong P, Tanaporn U, Sopacha A, Panomkorn C, Nitsara K, Wanilada R (2020) Bacterial analysis in the early developmental stages of the black tiger shrimp (Penaeus monodon). Sci Rep 10:4896

10. Liu B, Bo L, Qunlan Z, Cunxin S, Changyou S, Huimin Z, Zhenfei Y, Shana F (2020) Patterns of bacterial community composition and diversity following the embryonic development stages of Macrobrachium rosenbergii. Aquacult Rep 17:100372

11. Zheng D, Liwinski T, Elinav E (2020) Interaction between microbiota and immunity in health and disease. Cell Res 30:492-506

12. Wang H, Huang J, Wang P, Li T (2020) Insights into the microbiota of larval and postlarval Pacific white shrimp (Penaeus vannamei) along early developmental stages: a case in pond level. Mol Genet Genom 295:1517-1528

13. Patil PK, Vinay TN, Ghate SD, Baskaran V, Avunje S (2021) 16S rRNA gene diversity and gut microbial composition of the Indian white shrimp (Penaeus indicus). Antonie van Leeuwenhoek, https://doi.org/10.1007/s10482-021-01658-9

14. FAO (2020) The State of World Fisheries and Aquaculture 2020. Sustainability in action. Rome. https://doi.org/10.4060/ca9229en

15. Naylor1 RL, Hardy RW, Buschmann AH, Bush SR, Cao L, Klinger DH, Little DC, Lubchenco J, Shumway SE, Max T (2021) A 20-year retrospective review of global aquaculture. Nature 591:551563

16. Sajeela KA, Gopalakrishnan A, Basheer VS, Mandal A, Bineesh KK, Grinson G, Gopakumar SD (2019) New insights from nuclear and mitochondrial markers on the genetic diversity and structure of the Indian white shrimp Fenneropenaeus indicus among the marginal seas in the Indian Ocean. Mol Phylogenet Evol 136:53-64

17. Vijayan KK (2019) Domestication and genetic improvement of Indian white shrimp, Penaeus indicus: A complimentary native option to exotic Penaeus vannamei. J Coast Res 86:270-276 
18. Schloss PD, Westcott SL, Raybin T, Hall JR, Hartmann M (2009) Introducing Mothur: open-source. platform-independent, community-supported software for describing and comparing microbial communities. Appl Environ Microbiol 75:7537-7541

19. Heberle H, Meirelles GV, da-Silva FR, Telles GP, Minghim R (2015) InteractiVenn: a web-based tool for the analysis of sets through Venn diagrams. BMC Bioinfo 16:169

20. Dhariwal A, Chong J, Habib S, King I, Agellon LB, Xia J (2017) MicrobiomeAnalyst: a web-based tool for comprehensive statistical, visual and meta-analysis of microbiome data. Nucleic Acids Res 45:180-188

21. Hansen GH, Olafsen JA (1989) Bacterial Colonization of Cod (Gadus morhua L.) and halibut (Hippoglossus hippoglossus) eggs in marine aquaculture. Appl Environ Microbiol 55:1435-1446

22. Zhang Z, Liu J, Jin X, Liu C, Fan C, Guo L, Liang Y, Zheng J, Peng N (2020) Developmental, dietary, and geographical impacts on gut microbiota of red swamp crayfish (Procambarus clarkii). Microorganisms 8:1376

23. Ooi MC, Goulden EF, Smith GG, Nowak BF, Bridle AR (2017) Developmental and gut-related changes to microbiomes of the cultured juvenile spiny lobster Panulirus ornatus. FEMS Microbiol Ecol 93:fix159

24. Klindworth A, Elmar P, Timmy S, Jorg P, Christian Q, Matthias H, Frank OG (2013) Evaluation of general $16 \mathrm{~S}$ ribosomal RNA gene PCR primers for classical and next-generation sequencing-based diversity studies. Nucleic Acid Res 4(1):101093

25. Cheung MK, Yip HY, Nong W, Law PTW, Chu KH, Kwan HS, Hui JHL (2015) Rapid change of microbiota diversity in the gut but not the hepatopancreas during gonadal development of the new shrimp model Neocaridina denticulata. Mar Biotech 17:811-819

26. Liu H, Wang L, Liu M, Wang B, Jiang K, Ma S, Li Q (2011) The intestinal microbial diversity in Chinese shrimp (Fenneropenaeus chinensis) as determined by PCR-DGGE and clone library analyses. Aquaculture 317:32-36

27. Rungrassamee W, Klanchui A, Maibunkaew S, Karoonuthaisiri N (2016) Bacterial dynamics in intestines of the black tiger shrimp and the Pacific white shrimp during Vibrio harveyi exposure. $J$ Invert Pathol 133:12-19

28. Rungrassamee W, Klanchui A, Chaiyapechara S, Maibunkaew S, Tangphatsornruang S (2013) Bacterial population in intestines of the Black Tiger Shrimp (Penaeus monodon) under different growth stages. PLoS ONE 8(4):e60802

29. Rungrassamee W, Klanchui A, Maibunkaew S, Chaiyapechara S, Jiravanichpaisal P, Karoonuthaisiri N (2014) Characterization of intestinal bacteria in wild and domesticated adult black tiger shrimp (Penaeus monodon). PLoS ONE 9(3):e91853

30. Turnes MSG, Hay ME, Fenical W (1989) Symbiotic marine bacteria chemically defend crustacean embryos from a pathogenic fungus. Science 246(4926):116-118

31. Luo H, Moran MA (2014) Evolutionary ecology of the marine Roseobacter clade. Microbiol Mol Biol Rev 78(4):573-587 
32. Curiel FB, Ramirez-Pueblab ST, Ringøc E, Escobar-Zepedad A, Godoy-Lozanod E, Vazquez-Duhalte R, Sanchez-Floresd A, Vianaf MT (2018) Effects of extruded aquafeed on growth performance and gut microbiome of juvenile Totoaba macdonaldi. Anim Feed Sci Technol 245:91-103

33. Yao Z, Yang K, Huang L, Huang X, Qiuqian L, Wang L, Zhang D (2018) Disease outbreak accompanies the dispersive structure of shrimp gut bacterial community with a simple core microbiota. AMB Expr 8:120

34. Crenn K, Duffieux D, Jeanthon C (2018) Bacterial epibiotic communities of ubiquitous and abundant marine diatoms are distinct in short- and long-term associations. Front Microbiol 9:2879-2879

35. Lim Y, Innam K, Cho JC (2020) Genome characteristics of Kordia antarctica IMCC3317T and comparative genome analysis of the genus Kordia. Sci Rep 10:14715

36. Chen B, Du K, Sun C, Vimalanathan A, Liang X, Li Y, Wang B, Lu X, Li L, Shao Y (2018) Gut bacterial and fungal communities of the domesticated silkworm (Bombyx mori) and wild mulberry-feeding relatives. ISME J 12:2252-2262

37. Hammer TJ, McMillan WO, Fierer N (2014) Metamorphosis of a butterfly-associated bacterial community. PLoS ONE 9:0086995

\section{Tables}

Table 1. Alpha diversity indices for the sequence libraries of $P$. indicus larval stages

\begin{tabular}{cccc}
\hline Developmental stage & Chao & ACE & Shannon \\
\hline Egg & $579.86 \pm 22.25$ & $564.56 \pm 11.72$ & $3.81 \pm 0.02$ \\
Nauplii & $662.61 \pm 20.19$ & $789.84 \pm 11.93$ & $4.29 \pm 0.12$ \\
Zoea & $830.5 \pm 16.87$ & $816.72 \pm 14.01$ & $4.17 \pm 0.21$ \\
Mysis & $841.22 \pm 16.82$ & $828.70 \pm 14.17$ & $4.37 \pm 0.17$ \\
PL1 & $627.08 \pm 26.06$ & $594.20 \pm 11.51$ & $3.56 \pm 0.42$ \\
PL6 & $603.49 \pm 25.50$ & $586.23 \pm 11.14$ & $3.38 \pm 0.47$ \\
PL12 & $706.53 \pm 33.85$ & $816.72 \pm 12.44$ & $3.35 \pm 0.32$ \\
\hline
\end{tabular}

Table 2. Most abundant microbiota at early developmental stages of $P$. indicus. 


\begin{tabular}{|c|c|c|c|c|}
\hline $\begin{array}{c}\text { Shrimp } \\
\text { developmental stage }\end{array}$ & Phylum & $\begin{array}{c}\text { Relative } \\
\text { abundance (\%) }\end{array}$ & Genus & $\begin{array}{c}\text { Relative } \\
\text { abundance } \\
\text { (\%) }\end{array}$ \\
\hline \multirow[t]{16}{*}{ Egg } & \multirow[t]{8}{*}{ Proteobacteria } & \multirow[t]{8}{*}{51.20} & Alteromonas & 19.67 \\
\hline & & & Alteromonadaceae_unclassified & 7.65 \\
\hline & & & Rhodobacteraceae_unclassified & 4.62 \\
\hline & & & Nautella & 4.60 \\
\hline & & & Kordiimonas & 3.03 \\
\hline & & & Pseudoalteromonas & 2.92 \\
\hline & & & Shimia & 2.49 \\
\hline & & & Vibrio & 1.36 \\
\hline & \multirow[t]{5}{*}{ Bacteroidota } & \multirow[t]{5}{*}{35.15} & Winogradskyella & 12.64 \\
\hline & & & Mesoflavibacter & 10.97 \\
\hline & & & NS11-12_marine_group_ge & 3.74 \\
\hline & & & Flavobacteriaceae_unclassified & 2.76 \\
\hline & & & Muricauda & 2.46 \\
\hline & Verrucomicrobiota & 5.52 & Haloferula & 5.23 \\
\hline & Firmicutes & 2.51 & * & \\
\hline & Myxococcota & 1.99 & * & \\
\hline \multirow[t]{17}{*}{ Nauplii } & \multirow[t]{11}{*}{ Proteobacteria } & \multirow[t]{11}{*}{71.234} & Alteromonas & 12.55 \\
\hline & & & Nautella & 9.02 \\
\hline & & & Vibrio & 5.64 \\
\hline & & & Pseudoalteromonas & 5.42 \\
\hline & & & Vibrionaceae_unclassified & 3.53 \\
\hline & & & Rhodobacteraceae_unclassified & 3.42 \\
\hline & & & Thalassotalea & 3.20 \\
\hline & & & Hyphomonadaceae_unclassified & 3.17 \\
\hline & & & Pseudomonas & 3.00 \\
\hline & & & Kordiimonas & 2.56 \\
\hline & & & Shimia & 2.50 \\
\hline & \multirow[t]{3}{*}{ Bacteroidota } & \multirow[t]{3}{*}{21.81} & Tenacibaculum & 5.94 \\
\hline & & & Flavobacteriaceae_unclassified & 2.75 \\
\hline & & & Mesoflavibacter & 2.53 \\
\hline & Firmicutes & 1.59 & * & \\
\hline & Actinobacteriota & 1.08 & * & \\
\hline & Desulfobacterota & 0.83 & * & \\
\hline \multirow[t]{14}{*}{ Zoea } & \multirow[t]{8}{*}{ Proteobacteria } & \multirow[t]{8}{*}{49.38} & Rhodobacteraceae_unclassified & 6.44 \\
\hline & & & Gammaproteobacteria_unclassified & 4.90 \\
\hline & & & Shimia & 4.06 \\
\hline & & & Alteromonas & 3.88 \\
\hline & & & Pseudoalteromonas & 3.29 \\
\hline & & & Neptuniibacter & 2.45 \\
\hline & & & Marivita & 2.35 \\
\hline & & & Nautella & 2.34 \\
\hline & \multirow[t]{3}{*}{ Bacteroidota } & \multirow[t]{3}{*}{22.33} & Saprospiraceae_unclassified & 6.92 \\
\hline & & & Tenacibaculum & 4.02 \\
\hline & & & Flavobacteriaceae_unclassified & 1.51 \\
\hline & Verrucomicrobiota & 14.06 & Roseibacillus & 12.85 \\
\hline & Planctomycetota & 8.73 & Rubinisphaeraceae_unclassified & 6.74 \\
\hline & Margulisbacteria & 1.67 & Margulisbacteria_ge & 1.68 \\
\hline \multirow[t]{3}{*}{ Mysis } & \multirow[t]{3}{*}{ Proteobacteria } & \multirow[t]{3}{*}{51.62} & Pseudoalteromonas & 6.40 \\
\hline & & & Rhodobacteraceae_unclassified & 5.90 \\
\hline & & & Kordiimonas & 5.24 \\
\hline
\end{tabular}




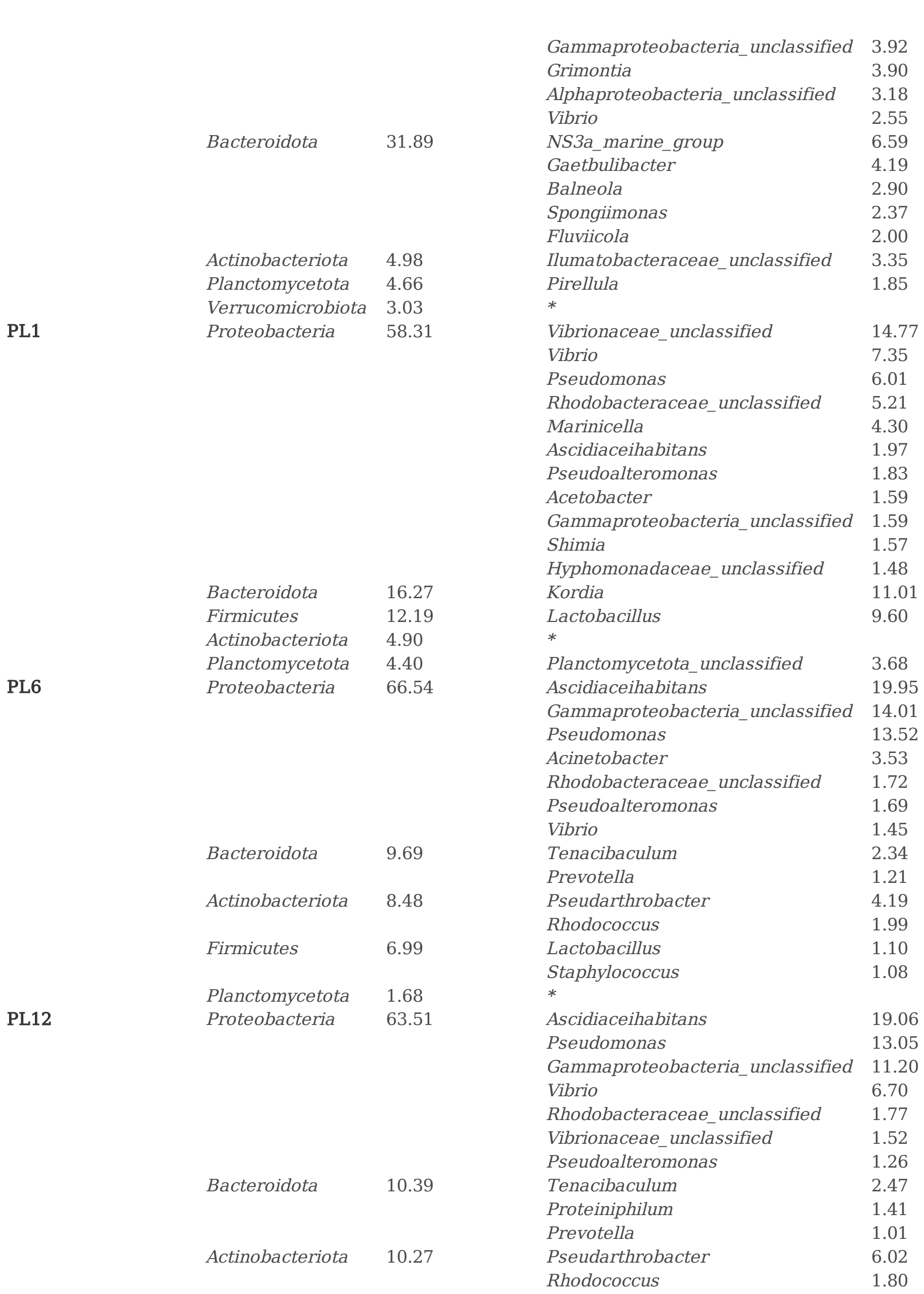


Note: $\left.{ }^{*}\right)$; Represented by many genera in lower abundance.

Figures

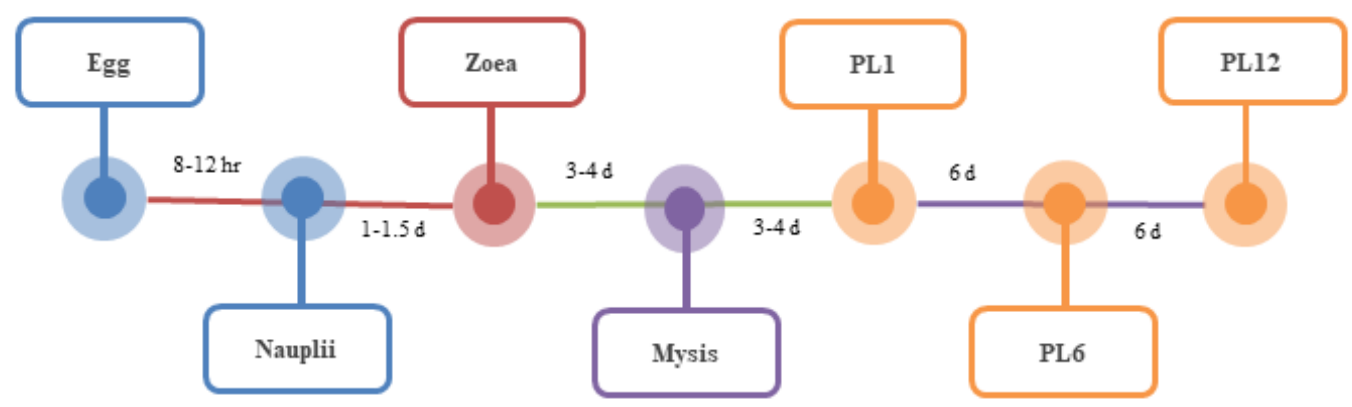

Fig 1

\section{Figure 1}

Schematic diagram showing the sampling points during $P$. indicus hatchery cycle. 


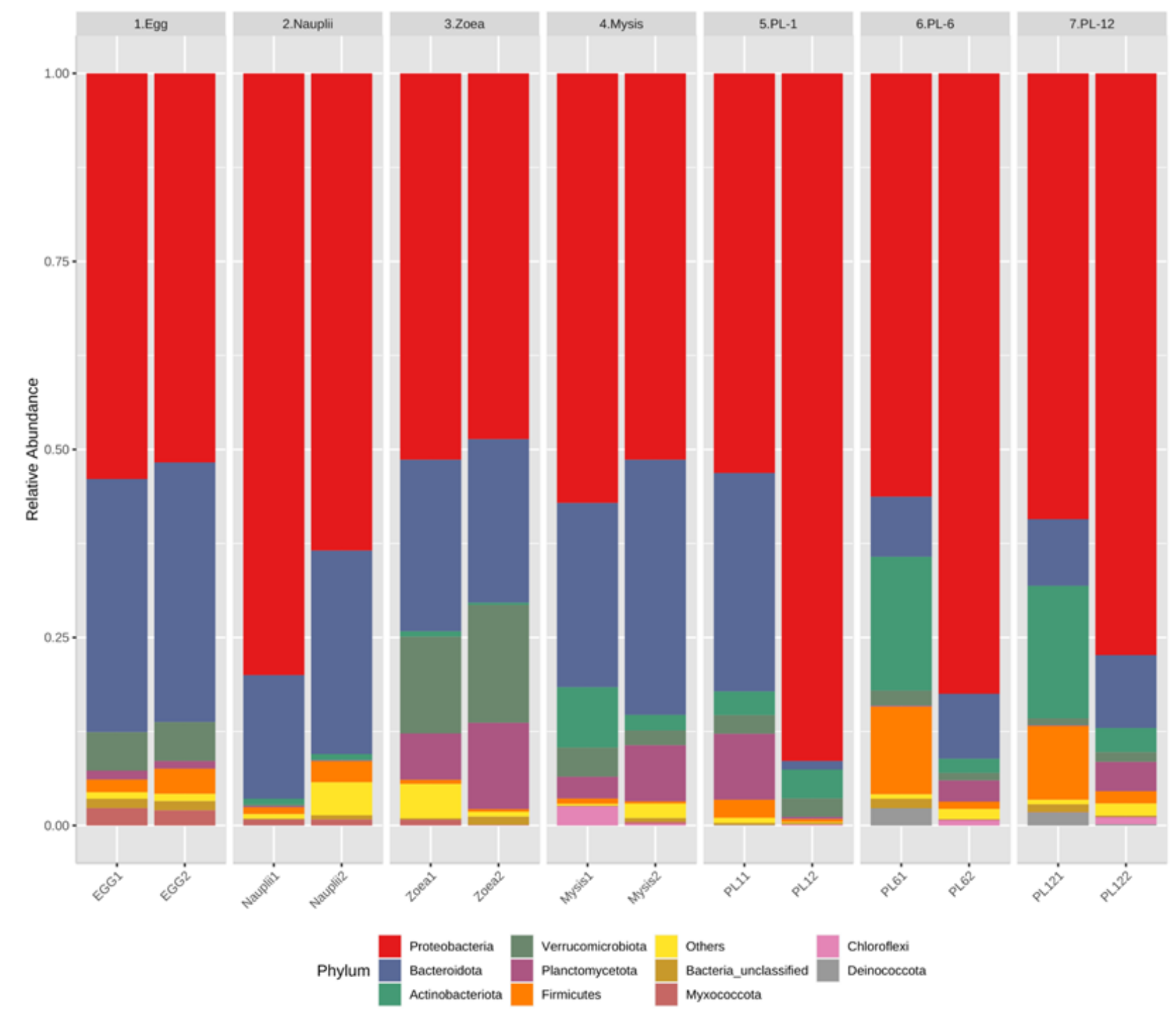

Figure 2

Distribution of bacterial phyla (Relative abundance) in various developmental stages of $P$. indicus. 

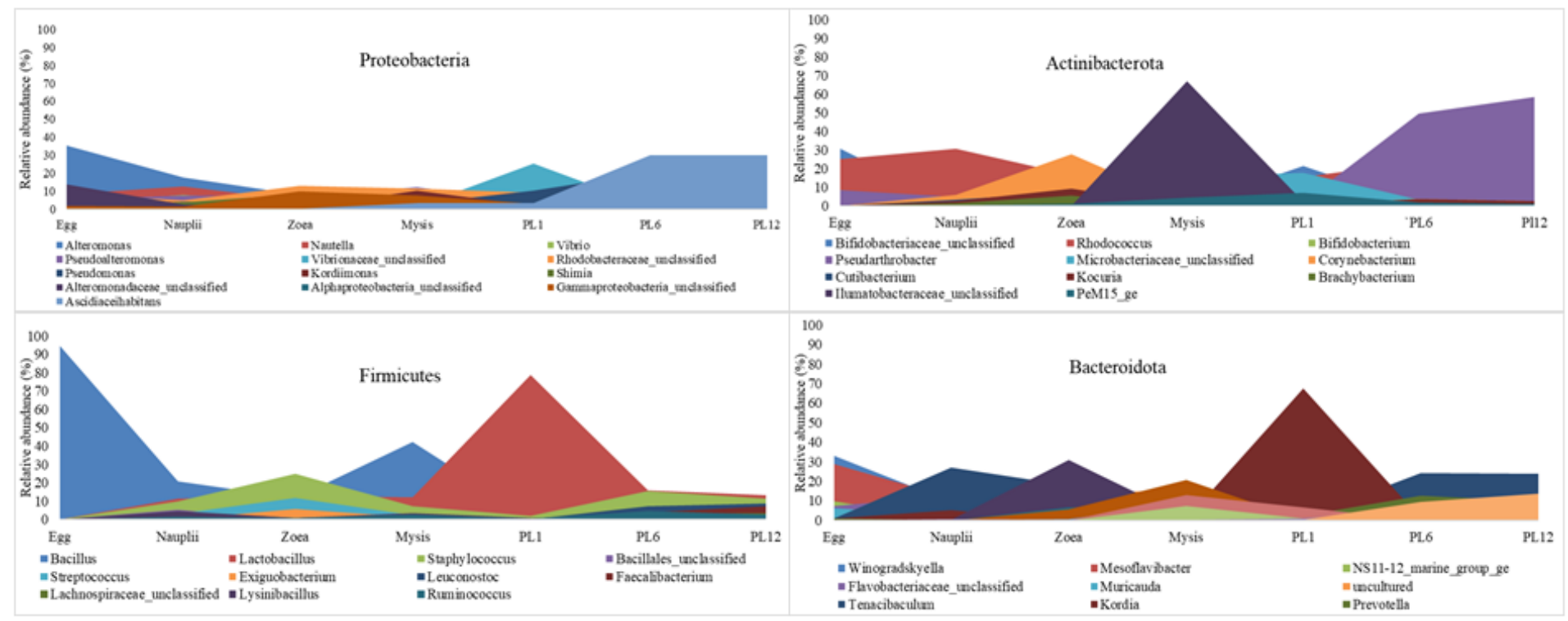

Fig 3

\section{Figure 3}

Relative abundance of dominant genus under major phylum in various developmental stages of $P$. indicus. 


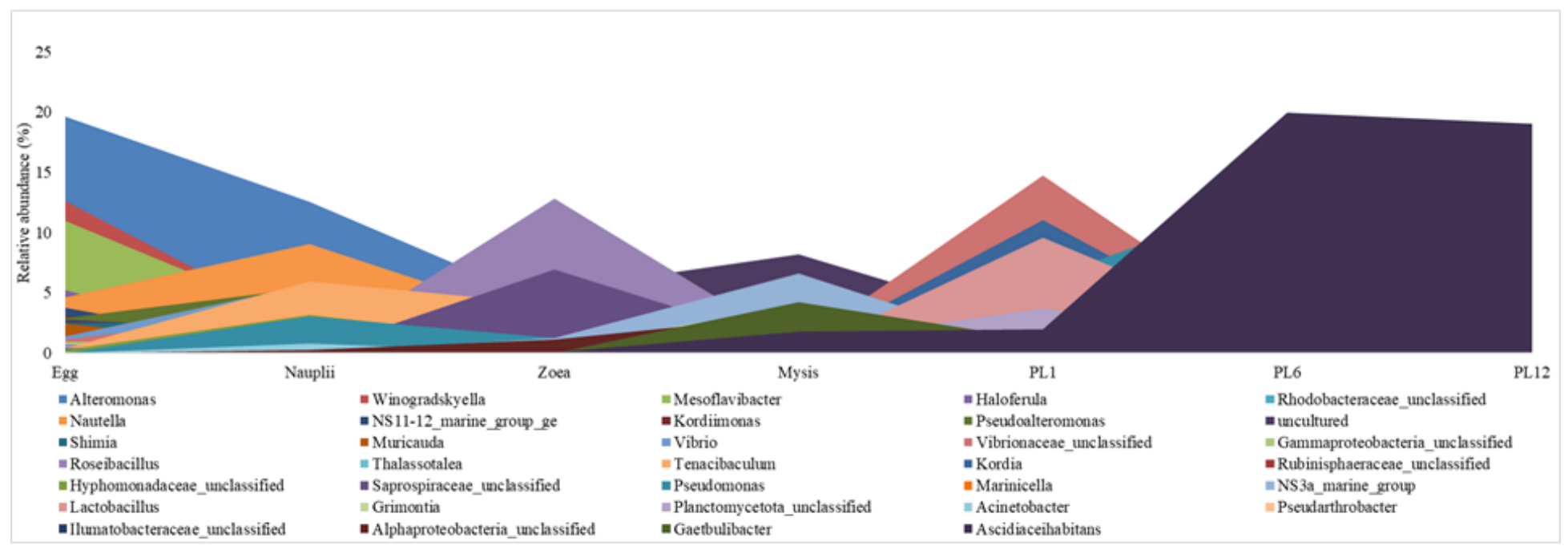

Fig 4

Figure 4

Dynamics of dominant genera in in various developmental stages of $P$. indicus. 


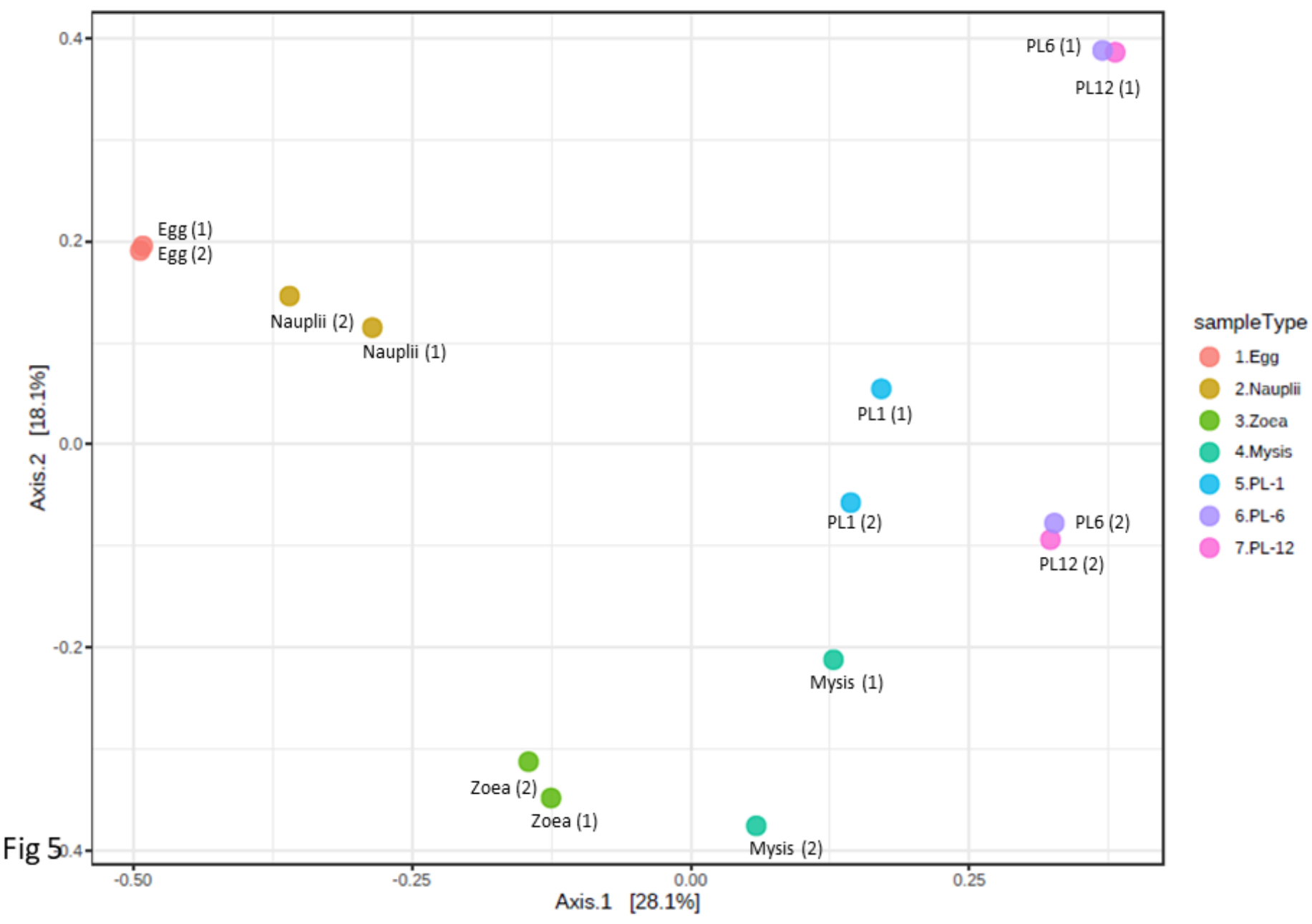

Fig 5

\section{Figure 5}

Principal coordinates analysis (PCOA) of microbial communities associated with various developmental stages of $P$. indicus in two full sib families (Egg, Nauplii, Zoea, Mysis, PL1, PL6, PL12). 


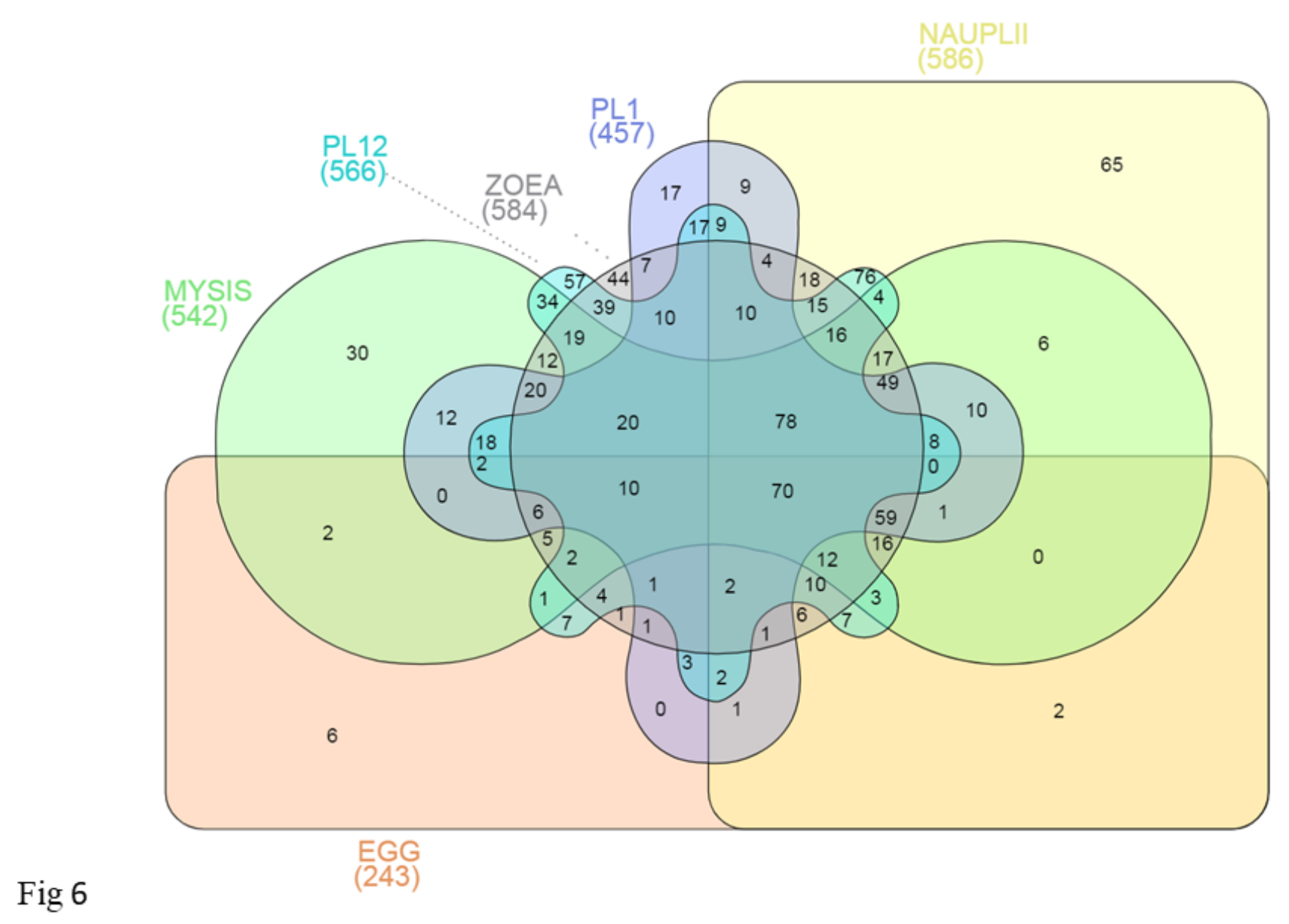

Figure 6

Venn diagram depicting the shared and unique genera among various developmental stages of $P$. indicus.

\section{Supplementary Files}

This is a list of supplementary files associated with this preprint. Click to download.

- SupplementaryFigS1.pdf

- SupplementaryTaxonomy.xlsx 